\title{
NON-LUMINESCENT DISEXCITATION OF F-CENTER PAIRS BY EXCHANGE-SOFTENED VIBRATIONAL MODES
}

\author{
C. JACCARD and M. AEGERTER \\ Institut de Physique de l'Université, 2000 Neuchâtel, Switzerland
}

Received 18 April 1973

\begin{abstract}
The non-luminescent and spin-dependent disexcitation process observed in F-center pairs in alkali halides at low temperature is explained by a covalent bond within the pair. Exchange effects give a negative contribution to the lattice potential energy. If the pair separation is small, local modes become unstable and spontaneous lattice distortions bring back the pair in its ground state.
\end{abstract}

Alcali halide crystals with a high concentration of $F$ centers show at low temperature a strong variation of the luminescent quantum yield under an applied static magnetic field $[1,2]$ which allows optical detection of electronic and nuclear magnetic resonances $[3,4]$. These phenomena are explained by interaction of pairs of neighbouring centers, in which the electronic disexcitation path (radiative or non-radiative) depends on the symmetry of the two-spin wave function. According to an hypothesis put forward by Markham et al. [5] and resumed by Lüty [5] in order to explain the correlation observed between the $F$ and the $F^{\prime}$ band intensities, the electron of the excited member of a pair shifts non-radiatively to the other member to form an $F^{\prime}$ center and then comes back to its ground state by a tunnel effect. The necessity for the $F^{\prime}$ center to be in a singlet spin state sets the condition for this process, consistent with most of the observations. This mechanism, being spin dependent, should respond to EPR and if it is the only one, the relative variation of the $F^{\prime}$ concentration should be of the order of the relative luminescence variation $\left(>10^{-2}\right)$. But with well separated pairs [3], the $\mathrm{F}^{\prime}$ absorption dependence on EPR is much smaller than $10^{-3}$ [7]. This suggests another spin dependent mechanism for non radiative disexcitation, which has nothing to do with transient $\mathrm{F}^{\prime}$ centers. In this paper we develope qualitatively the following hypothesis: Provided a suitable spin state, a covalent bond is formed between the two members of the pair; the exchange "softens" certain localized vibrational lattice modes, up to the point to make them unstable, so that even at zero temperature the system shifts to its ground state only by tumbling down vibrational states.
We consider a pair of F centers located at sites $a$ and $b$ (separation $R$ ). The first one is in its relaxed excited state and the second one in its ground state. A local vibrational mode (frequency $\omega$, amplitude $q$ with $\left.\left\langle q^{2}\right\rangle_{T=0}=1\right)$ mixes to the electronic state $\psi_{0}$ another one $\psi_{1}$, having an energy higher by $\delta$. Up to the second order, the $q$ dependent wave function and its energy take the form

$\psi=\psi_{0}\left(1-\frac{1}{2} w^{2} q^{2}\right)-w q \psi_{1}+\ldots$,

$E=E_{0}-\delta w^{2} q^{2}+\ldots$,

the $\psi_{1}$ admixture being then $w^{2}$. With this wave function, the total energy of the pair is calculated according to the Heitler-London scheme, while assuming that the perturbation of the second center at $b$ is negligible $($ state $\varphi)$. The result is a function of the pair $\operatorname{spin}(S=0$ or 1) and of $q$, with an exchange energy $J_{0}$ for $q=0$. The term in $w^{2} q^{2}$, including the vibrational potential energy, is relevant for the stability and takes the form

$$
\begin{aligned}
& \frac{1}{2} \frac{\mathrm{d}^{2} E}{\mathrm{~d}(w q)^{2}}=\frac{\hbar \omega}{4 w^{2}}+\left\{-\delta+\left\langle\psi_{1}\left|V_{\mathrm{b}}\right| \psi_{1}\right\rangle-\left\langle\psi_{0}\left|V_{\mathrm{b}}\right| \psi_{0}\right\rangle\right. \\
& \left.\quad+\left\langle\psi_{1} \varphi\left|\frac{e^{2}}{r}\right| \psi_{1} \varphi\right\rangle-\left\langle\psi_{0} \varphi\left|\frac{e^{2}}{r}\right| \psi_{0} \varphi\right\rangle\right\}+\left(S-\frac{1}{2}\right)\left(J_{1}-J_{0}\right)
\end{aligned}
$$

$J_{1}$ is the exchange energy for the $a$-center in a pure $\psi_{1}$ state and $V_{\mathrm{b}}$ is the local potential at site $b$. The expression in the parenthesis is independent of the total spin $S$ and is certainly negative because the electron clouds screen the electrostatic interaction between the ions and thereby soften the local mode, reducing its frequency to $\omega^{\prime}<\omega$. The last, spin dependent term induces a softening only for the singlet state $S=0$, and this can lead to an instability of the mode, i.e. a nega- 


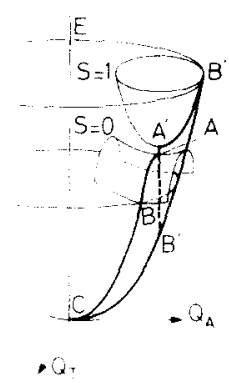

Fig. 1. Configuration diagram for the energy of the triplet and singlet relaxed excited states and for the ground state (see text).

tive frequency, provided the two following conditions are satisfied:

1) $J_{1}>J_{0}$ : this should be the case since the higher lying state $\psi_{1}$ extends farther in the lattice than the lower lying state $\psi_{0}$.

2) The local mode should couple strongly the two states, so that the admixture is large:

$w^{2}>\hbar \omega^{\prime} / 2\left(J_{1}-J_{0}\right)$.

These conditions are satisfied if the separation of the pair is smaller than a critical value $R_{\mathrm{c}}$. This parameter has been estimated with properly scaled hydrogenlike wave functions, approximating the excited states $\psi_{0}$ and $\psi_{1}$ by $2 s$ - and 2 p-functions, respectively. They should describe reasonably well the $\mathrm{F}$ center at large distance. For the ground state, a 1 s-function can be used and the integrals can be calculated from

Roothaan's formulae [9]; an alternative way is to assume a delta function, and the evaluation is then straightforward. In both cases, one finds a critical radius of about $30-40 \AA$, provided the admixture is of the order of 0.1 . This agrees with the model proposed by Bogan and Fitchen [8]. The estimated critical radius is within a factor of 2 or 3 of the value of $80 \AA$ obtained from the luminescent behaviour in a magnetic field [2]. However, according to a recent work by F.S. Ham (private communication), the considered states are not purely electronic but vibronic, and taking this into account would give a better description of the lattice with the electrons.

The cycle of an optically excited center, in the pres- ence of a neighbour within the critical distance, can be depicted in a configuration diagram (fig. 1), for a non softening mode $A$ and a softening mode $T$. The former expresses the relacation between the ground state $F_{0}(\mathrm{ls})$ and a relaxed excited state $\widetilde{F}^{*}(2 \mathrm{~s})$, and the latter couples $\widetilde{F}^{*}(2 s)$ to a higher lying state e.g. $\widetilde{F}^{*}(2 \mathrm{p})$.

The instability of the $T$ mode for the spin singlet produces a saddle-shaped energy surface, on which the system glides until it meets the energy surface of the ground state $(\mathrm{ABC})$. For the spin triplet, the energy surface has always a minimum, from which the ground state is reached either by a vertical radiative transition $\left(A^{\prime} B^{\prime} C\right)$ or through a highly excited vibrational state at high temperature $\left(A^{\prime} B^{\prime \prime} C\right)$. The effect of the neighbouring centre is to decrease the curvature of the energy surface preferentially for the singlet spin state, and this is enough to change the luminescent yield of the pair, in agreement with the observations. At short distance, the ground state might show a secondary potential well corresponding to the pair " $\mathrm{F}$ ' + vacancy" into which the system can glide and get trapped momentarily, until it reaches point $\mathrm{C}$ by tunnelling through the potential wall.

We are indebted to Professor F. Bassani for valuable discussions and to the Swiss National Foundation for support of this work.

\section{References}

[1] F. Porret, F. Lüty, Phys. Lett. 26 (1971) 843.

[2] C. Jaccard et al., Phys. Stat. Sol. (b) 50 (1972) 187.

[3] Y. Ruedin et al., Phys. Stat. Sol. (b) $S 4$ (1972) 565, 55 (1973) 215.

[4] P.A. Schnegg, C. Jaccard and M. Aegerter, Phys. Lett. 42A (1973) 369.

[5] J.J. Markham, R.T. Platt and I.L. Mador, Phys. Rev. 92 (1953) 957.

[6] F. Lüty, Halbleiterphysik, Vol. VI, 238 (1961) (Viehweg, Braunschweig).

[7] P.A. Schnegg et al., (to be published).

[8] L.D. Bogan and D.B. Fitchen, Phys. Rev. B, 1 (1970) 4122.

[9] C.C.J. Roothaan, J. Chem Phys. 19 (1951) 1445. 